\title{
A barrier against reactive oxygen species: chitosan/acellular dermal matrix scaffold enhances stem cell retention and improves cutaneous wound healing
}

Wei Lin ${ }^{1,2}$, Xiaoyang Qi ${ }^{3}$, Wenjing Guo ${ }^{4}$, Danyang Liang ${ }^{1,2}$, Heting Chen ${ }^{1,2}$, Baoping Lin ${ }^{1,2}$ and Xiaoyuan Deng ${ }^{1,2^{*}}$ (D)

\begin{abstract}
Background: Stem cell therapies have gained great attention for providing novel solutions for treatment of various injuries and diseases due to stem cells' self-renewal, ability to differentiate into various cell types, and favorite paracrine function. Nevertheless, the low retention of transplanted stem cell still limits their clinical applications such as in wound healing in view of an induced harsh microenvironment rich in reactive oxygen species (ROS) during inflammatory reactions.
\end{abstract}

Methods: Herein, a novel chitosan/acellular dermal matrix (CHS/ADM) stem cell delivery system is developed, which is of great ROS scavenging activity and significantly attenuates inflammatory response.

Result: Under ROS microenvironment, this stem cell delivery system acts as a barrier, effectively scavenging an amount of ROS and protecting mesenchymal stem cells (MSCS) from the oxidative stress. It notably regulates intracellular ROS level in MSCs and reduces ROS-induced cellular death. Most importantly, such MSCs delivery system significantly enhances in vivo transplanted stem cell retention, promotes the vessel growth, and accelerates wound healing.

Conclusions: This novel delivery system, which overcomes the limitations of conventional plain collagen-based delivery system in lacking of ROS-environmental responsive mechanisms, demonstrates a great potential use in stem cell therapies in wound healing.

Keywords: Chitosan/Acellular dermal matrix scaffold, ROS barrier in inflammatory response, Stem cell retention, Wound healing

\section{Background}

There is a great potential for stem cell-based therapies to treat a myriad of diseases and injuries by means of stem cells' ability to modulate immune or inflammatory responses through releasing trophic factors [1-3]. However,

\footnotetext{
* Correspondence: xiaoyuandeng@scnu.edu.cn

${ }^{1}$ MOE Key Laboratory of Laser Life Science, College of Biophotonics \& Institute of Laser Life Science, South China Normal University, Guangzhou 510631, China

${ }^{2}$ Guangdong Provincial Key Laboratory of Laser Life Science, College of Biophotonics, South China Normal University, Guangzhou 510631, China Full list of author information is available at the end of the article
}

the low engraftment rates and low retention of transplanted stem cells, caused by lacking of an appropriate 3D supporting matrix for efficient stem cells delivery as well as survival microenvironment providing for stem cells, are still the critical factors that limit their clinical applications in wound healing [4]. To address this issue, scaffolds have been proposed as an effective strategy to deliver stem cells to the desired site of repair, which offer stem cells a structural support and a bio-functionally living microenvironment for cell adhesion, migration and proliferation [5]. In recent years, two major scaffold materials have been

C C The Author(s). 2020 Open Access This article is licensed under a Creative Commons Attribution 4.0 International License, which permits use, sharing, adaptation, distribution and reproduction in any medium or format, as long as you give appropriate credit to the original author(s) and the source, provide a link to the Creative Commons licence, and indicate if changes were made. The images or other third party material in this article are included in the article's Creative Commons licence, unless indicated otherwise in a credit line to the material. If material is not included in the article's Creative Commons licence and your intended use is not permitted by statutory regulation or exceeds the permitted use, you will need to obtain permission directly from the copyright holder. To view a copy of this licence, visit http://creativecommons.org/licenses/by/4.0/ The Creative Commons Public Domain Dedication waiver (http://creativecommons.org/publicdomain/zero/1.0/) applies to the data made available in this article, unless otherwise stated in a credit line to the data. 
investigated, the artificial and natural materials. Natural materials usually have better biocompatibility and biodegradability compared to artificial materials. Natural collagen, which has excellent biocompatibility and enzymatic degradability, becomes one of the most popular biomaterials being widely explored as implantable scaffold $[6,7]$. Acellular dermal matrix (ADM), derived from dermal extracellular matrix (ECM) of human or animal skin by decellularization process, is so far the popular applicable collagen-based bioscaffold. It is mainly composed of collagen proteins, some other remaining functional proteins such as fibronectin, laminin, and small amount of glycoproteins, glycosaminoglycans, proteoglycans, etc. ADM has a completely analogous structure and components to natural dermal skin; therefore, it has remarkable biocompatibility and biodegradability. Also, ADM is capable of providing a 3D matrix with favorable bio-structural and bio-functional properties like mechanical strength for mesenchymal stem cells (MSCs) survival. So ADM has been used as a powerful vehicle for stem cell delivery to enhance skin regeneration and tissue defect $[8,9]$.

However, due to the degradation of collagen, the collagen-based stem cell delivery system has shortcomings in coping with the inflammatory response in wound healing. Wound healing is a highly dynamic process which involves complex interactions of subtypes of infiltrating leukocyte and various resident cells [10, 11]. Infiltrating polymorphonuclear leukocytes, such as neutrophils, are the principal cellular components acting on the inflammatory response at the wound site [12, 13]. Reactive oxygen species (ROS) such as hydroxyl $(\cdot \mathrm{HO})$, superoxide anion radicals $\left(\mathrm{O}_{2}{ }^{-}\right)$, and hydrogen peroxide $\left(\mathrm{H}_{2} \mathrm{O}_{2}\right)$ are released by neutrophils to eliminate infectious threats $[14,15]$. In the collagen-based stem cell delivery system, more neutrophils are supposed to accumulate in wound site along with the biodegradation process of collagen, as the generated peptide fragments of collagen were found as chemotactic factors and activators of neutrophils [16]. The activation of neutrophils results in several distinct morphological and metabolic events, all leading to phagocytosis and degradation of foreign substances to generate overabundant ROS [17-24]. Such overproduction of ROS cause an imbalance in the metabolism of reactive intermediates leading to oxidative stress damage in MSCs, and ultimately damage critical components of the implanted MSCs including their lipids, proteins, and DNA [25-32]. Hence, improving the ROS scavenging ability of the collagenbased scaffold to control the amount of ROS in microenvironment is of great concern, which will effectively avoid the hostile ROS-induced stem cells death, so as to preserve the essential paracrine factors secreted by stem cells to enhance tissue repairing capability.

Chitosan, a linear polymer of D-glucosamine with a $\beta-(1$, 4) linkage, with similar structure to the glycosaminoglycan portion of ECM, is a fascinating candidate for medical and pharmaceutical applications due to its unique biological properties [33-37]. It is completely biocompatible, bacteriostatic, hemostatic, and biodegradable of harmless substances and appears to act as a wound healing accelerator $[38,39]$.

Herein, we attempt to functionally modify ADM with chitosan to synthesize a novel chitosan/acellular dermal matrix (CHS/ADM) scaffold, which is applied to delivery MSCs for treating wound healing (Scheme 1). Chitosan is a polycationic material, while ADM is a type of polyanionic material that has a negatively charged surface, through the electrostatic interactions, chitosan will interact with ADM to form CHS/ADM. Under high ROS microenvironment which could be induced by collagen degradation in ADM, chitosan scavenges an amount of ROS and acts as an efficient barrier to protect MSCs from the oxidative stress. The ROS scavenging activity of chitosan are based on a hydrogen-donating ability of amine and hydroxyl group. The hydroxyl groups in the polysaccharide unit of the chitosan react with $\cdot \mathrm{OH}$ by the typical $\mathrm{H}$-abstraction reaction. In addition, the residual free amino groups $\mathrm{NH}_{2}$ in chitosan react with . $\mathrm{OH}$ to form stable macromolecule radicals $[15,40]$. Chitosan is a copolymer of glucosamine and $\mathrm{N}$-acetylglucosamine derived from the natural polymer chitin and could be metabolized in the body [41, 42]. Moreover, the degraded products of chitosan are nontoxic, nonimunogenic, and noncarcinogenic [43]. Therefore, this barrier against ROS would not have adverse reactions as it will ultimately degrade into harmless productions. This novel CHS/ADM stem cell delivery system is supposed to offer a favorable 3D matrix and reduce ROS-induced damage, which may significantly enhance the viable retention of implanted stem cells and further accelerate wound healing. To evaluate this, we first demonstrated the ROS scavenging capacity of the chitosan and then revealed the effectiveness of CHS/ADM scaffolds in decreasing the deterioration of MSCs survival by ROS. In particular, the effects of the scaffolds on neutrophils behavior and ROS release were assessed, which was followed by the investigation of viable retention of MSCs and therapeutic efficiency in an in vivo mouse excisional wound repair model.

\section{Method \\ Materials}

Chitosan ( $\geq 80 \%$ degree of deacetylation, M. W $40 \mathrm{w}-70$ w, YuanyeBio, Shanghai, China,), collagenase (BioFroxx, Guangzhou, China), and 2.5\% glutaraldehyde water solution was purchased from Shanghai Pharm. Co. (China). 0.25\% Dispase (Aoboxing, Beijing, China), cell counting kit-8 (CCK-8), C57BL/6 mouse bone marrow mesenchymal stem cells $\left(\mathrm{GFP}^{+}\right.$-MSCs, MUCMX-01101, Cyagen 


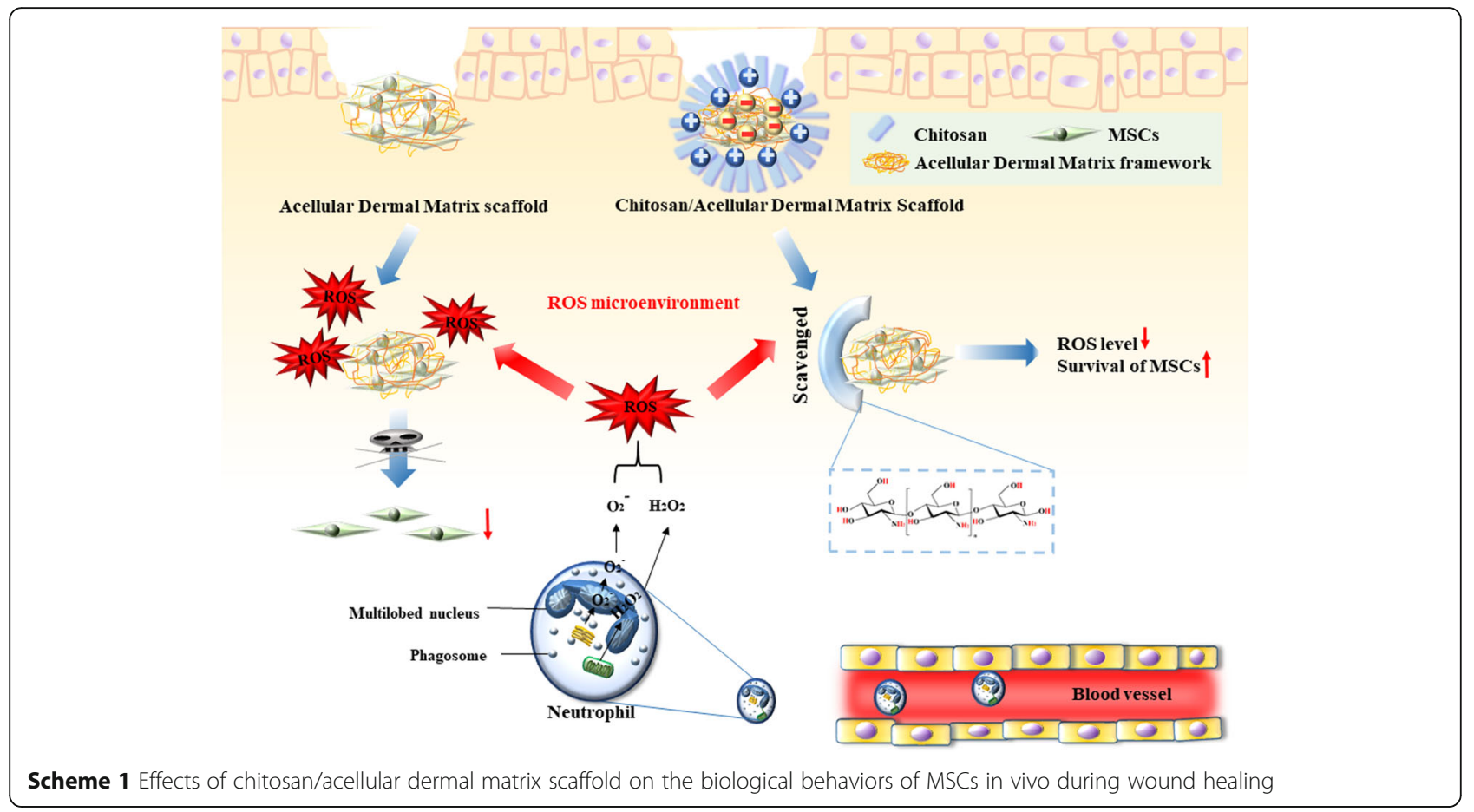

Biosciences, Inc.), and C57BL/6 mouse bone marrow mesenchymal stem cells (MSCs, MUBMX-01001, Cyagen Biosciences, Inc.) were also used along with OriCell mouse MSC growth medium and adipogenic and osteogenic differentiation medium (Cyagen Biosciences, Guangzhou, China). Dulbecco's modification of Eagle's medium (DMEM) and $0.25 \%$ trypsin-ethylenediaminetetraacetic acid (EDTA) were acquired from Gibco (Grand Island, NY). Other reagents were obtained from China National Medicine Corporation and used as received. Milli-Q water was used in all experiments.

Preparation and characterization of the CHS/ADM scaffold ADM was prepared with 5-week-old BALB/c mouse skin as described previously [8]. Briefly, the mice were euthanized, and the hair was shaved. The full-thickness skin was then harvested from the bare mice. Next, the skin tissues were immersed in $0.25 \%$ dispase at $4{ }^{\circ} \mathrm{C}$ for $48 \mathrm{~h}$ to remove the epidermis. The processed skin tissues were then washed using PBS and immersed in $0.3 \%$ Triton $\mathrm{X}-100,0.25 \%$ sodium deoxycholate, and $0.02 \%$ trypsin-EDTA with continuous shaking at $37^{\circ} \mathrm{C}$ for another $48 \mathrm{~h}$ in a horizontal shaker to remove the cellular components from the matrix. The acellular scaffolds were treated with a fat digestion solution of methanol and chloroform $(\mathrm{v} / \mathrm{v}=1: 1)$ for $2 \mathrm{~h}$ and washed with PBS. Finally, the scaffolds were cut into 8-mm-diameter round pieces for use. The prepared ADM was freezedried overnight firstly. Chitosan are positively charged in acidic medium, which was prepared as a $0.5 \%(\mathrm{w} / \mathrm{v})$ solution by means of $0.5 \mathrm{M}$ acetic acid solution. An ultrasonic cleaner was used to homogenize the chitosan solution. Further dilutions were necessary to obtain the various concentrations of stock solutions. The prepared $\mathrm{ADM}$ is initially denatured for $15 \mathrm{~min}$ at $60^{\circ} \mathrm{C}$ water solution and then a solution of chitosan thermostated at $40{ }^{\circ} \mathrm{C}$ is added to the prepared ADM. Chitosan was then self-assembled on the ADM scaffold to produce $\mathrm{CHS} /$ ADM scaffolds. Next, the scaffold was neutralized with the $1 \mathrm{M} \mathrm{NaOH}$ aqueous solution for $2 \mathrm{~h}$. Then, the samples were rinsed carefully with ultrapure water several times before lyophilization for $24 \mathrm{~h}$ to obtain the CHS/ ADM scaffold. Simultaneously, the ADM scaffold was immersed in PBS as the comparison group (ADM scaffold). Fourier transform infrared spectroscopy (FT-IR) of chitosan, ADM and CHS/ADM scaffolds were recorded by a Nicolet iS50 spectrometer (Thermo Scientific Inc., USA). The biodegradation stability of the ADM and CHS/ADM scaffolds was analyzed by treatment with 10 $\mathrm{mL}$ of PBS containing $100 \mu \mathrm{g} \mathrm{mL}^{-1}$ collagenase after weighing the initial weight (dry) of these scaffolds $\left(W_{0}\right)$. The biodegradation stability of ADM and CHS/ADM scaffolds in ROS environment was also studied with the same treatment. The degradation rates of these samples were explored at $24,48,72$, and $96 \mathrm{~h}$ at the temperature of $37^{\circ} \mathrm{C}$, replacing the fresh collagenase solution every $12 \mathrm{~h}$. The scaffolds were simultaneously removed from the degradation solution at a preset time point, carefully rinsed several times with deionized water, and freezedried after which the weight was recorded $\left(W_{t}\right)$. Finally, 
the following formula was used to calculate the degradation rate: $\mathrm{DR} \%=\left(W_{0}-W_{\mathrm{t}}\right) / W_{0} \times 100 \%$. All experiments were performed in triplicate.

The ADM, CHS/ADM scaffolds, and the scaffolds after degradation for $24 \mathrm{~h}$ were then fixed in $2.5 \%$ glutaraldehyde at $4{ }^{\circ} \mathrm{C}$ for $24 \mathrm{~h}$. Next, the scaffolds were dehydrated with graded ethanol $(30 \%, 50 \%, 75 \%, 90 \%$, and $100 \%$ ) for $15 \mathrm{~min}$ and immersed in hexamethylene diamine $(3 \times 15 \mathrm{~min})$. The dried scaffolds with a $\mathrm{CO}_{2}$ critical point were coated with a $30 \mathrm{~nm}$ gold layer for scanning electron microscopy (Zeiss Ultra 500; Carl Zeiss, Jena, Germany). Optimum SEM imaging parameters were as follows: extra high tension was set to $2 \mathrm{kV}$ and the operating distance was $5.4 \mathrm{~mm}$. The pore size and porosity distribution of the scaffolds were analyzed by Image-Pro Plus software.

\section{Cell culture}

The MSCs and $\mathrm{GFP}^{+}$-MSCs were cultured in OriCellTM mouse MSC Growth Medium (MUCMX-90011, Cyagen Biosciences, Inc.), added by $10 \%$ fetal bovine serum (FBS, Gibco) and $1 \%$ penicillin-streptomycin in a humidified incubator (Thermo Scientific Forma 3110, Thermo Fisher Scientific, Inc.) at $37^{\circ} \mathrm{C}, 5 \% \mathrm{CO}_{2}$. The ADM and CHS/ADM scaffolds were exposed to UV light overnight before the experiment. Round pieces of $\mathrm{ADM}$ and CHS/ADM scaffolds (8 $\mathrm{mm}$ in diameter) were acquired via biopsy punches and placed in a 48-well plate (one piece per well) with the papillary dermis side facing upward. ADM and CHS/ADM scaffolds were incubated overnight in fresh MSC Growth Medium, and then $1 \times 10^{5}$ MSCs were seeded on papillary dermis side of each ADM and CHS/ADM scaffolds and continued to culture in the MSC Growth Medium.

After $24 \mathrm{~h}$, the $\mathrm{GFP}^{+}$-MSCs on the ADM and CHS/ ADM scaffolds were photographed via a Carl Zeiss TPLSM 710 NLO META laser scanning microscope with a laser wavelength of $820 \mathrm{~nm}$. A cell counting experiment was also performed to quantitatively analyze the viability of MSCs on ADM and CHS/ADM. Briefly, the MSCs growing on the scaffold were enzymatically digested and resuspended into a single cell suspension. Cell counting was performed in cell counting plate after trypan blue staining $(n=6)$.

\section{Adipogenic and osteogenic differentiation assays}

After MSCs were cultured in 6-well dishes (control, chitosan, $\mathrm{H}_{2} \mathrm{O}_{2}, \mathrm{H}_{2} \mathrm{O}_{2}+$ chitosan) or on scaffolds (control, ADM, and CHS/ADM) for 1 day, the differentiation groups were treated with adipogenic and osteogenic medium for 14 and 21 days, respectively. Oil Red $\mathrm{O}$ staining and alizarin red staining were performed to verify adipocytes and osteoblasts, respectively. The morphologies of adipocytes and osteoblasts in the control (untreated), chitosan, $\mathrm{H}_{2} \mathrm{O}_{2}, \mathrm{H}_{2} \mathrm{O}_{2}$ +chitosan groups were photographed and then counted under microscope for statistical analyses. Total mRNA from the ADM and CHS/ADM scaffolds was isolated for RT-PCR analysis. Each experiment observed three random fields per sample and was repeated three times.

\section{Measurement of cell viability, adhesion, and intracellular ROS}

For the MSC viability assay, hydrogen peroxide $\left(\mathrm{H}_{2} \mathrm{O}_{2}\right)$ was used as the exogenous ROS source, and MSCs were treated with different concentrations of $\mathrm{H}_{2} \mathrm{O}_{2}$ and detected by the CCK- 8 assay. The MSCs treated with different chitosan concentrations were also detected by the CCK- 8 assay to investigate MSC viability in the ROS environment. Each sample was treated with $400 \mu \mathrm{L}$ of serum-free L-DMEM medium plus $40 \mu \mathrm{L}$ of CCK- 8 solution per well. After $6 \mathrm{~h}$ of incubation at $37^{\circ} \mathrm{C}$, the resulting production of water-soluble formazan dye was assayed at a wavelength of $450 \mathrm{~nm}$ by an EnspireTM multimode reader. Each sample was repeated three times in parallel. Simultaneously, calcein-AM/propidium iodide (Calcein-AM/PI) staining was used to evaluate the MSCs viability treated with different chitosan concentrations under ROS microenvironment. The excitation/ emission wavelength of AM is $480 \mathrm{~nm} / 452 \mathrm{~nm}$. The excitation/emission wavelength of PI is $535 \mathrm{~nm} / 617 \mathrm{~nm}$. For MSC-matrix adhesion assay, viable MSCs were suspended in medium to prepare cell suspensions and then were added to each well of a six-well plate and allowed to attach for $2 \mathrm{~h}$ at $37^{\circ} \mathrm{C}$ and $5 \% \mathrm{CO}_{2}$. Chitosan was used as ROS scavengers to evaluate their protection of MSCs from ROS. After adhesion, plates were carefully washed three times with PBS, and then five separate fields were counted under microscope. Each experiment was repeated at least three times. Subsequently, the intracellular ROS of the MSCs with the treatment of chitosan under the ROS environment were measured by immunofluorescence assay with dihydroethidium (DHE) staining.

MSCs $\left(1 \times 10^{5}\right)$ were cultured for $24 \mathrm{~h}$ and then seeded in CHS/ADM and ADM scaffolds for 24h (ADM scaffolds are as the comparison group). Calcein-AM/PI staining was used to evaluate the MSC survival activity seeded on scaffolds under ROS microenvironment. The CCK-8 assay was also performed to investigate MSC viability (\%) seeded on scaffolds in the ROS environment. In addition, the intracellular ROS of the MSCs-ADM and MSCs-CHS/ADM scaffolds under the ROS environment were measured. The fluorescence intensity was analyzed using ImageJ software. Each experiment was repeated at least three times. 
In vivo studies

Mouse model and implantation of MSCs seeded on the scaffold

The specific pathogen-free BALB/c mice (7-8 weeks) were supplied by the animal facility of Southern Medical University. All animal procedures were conducted in strict accordance with the Institutional Animal Care and Use Committee of the University of South China Normal University. The dorsal skin of each mouse was shaved and sterilized with $70 \%$ ethanol and iodine prior to surgery. Animals were anesthetized with an intraperitoneal injection of chloral hydrate $(400 \mathrm{mg} / \mathrm{kg}$, Fuchen, Tianjin, China). Once the mice were anesthetized, an 8$\mathrm{mm}$ biopsy punch was used to make an impression on the dorsum, and then the circular region of tissue was grabbed and pulled with forceps and excised with scissors to create a full-thickness wound. The wounded mice were randomly subjected to three groups $(n=6)$, which were separately treated as control (untreated wound), MSCs-ADM scaffold or MSCs-CHS/ADM scaffold. Checks for postoperative pain and complications were performed daily after the operation.

\section{Neutrophil infiltration and intracellular ROS}

The cutaneous wound repair areas were dissected after treatment with the MSCs-ADM and MSCs-CHS/ADM scaffolds after 1, 3, and 5 days. Then, the samples were inserted into paraffin and were perpendicularly sectioned into 3- $\mu \mathrm{m}$-thick longitudinal sections. The paraffin sections were stained with $\mathrm{CD} 11 \mathrm{~b}$ after deparaffinization and rehydration, and then washed with ultrapure water for histological analysis.

For immunofluorescence staining with dihydroethidium (DHE, Servicebio, Guangzhou, China), the frozen sections were slightly dried, a pap pen was used to make a circle around the tissue, and DHE was added to the circle followed by incubation at $37^{\circ} \mathrm{C}$ for $30 \mathrm{~min}$ in the dark. After washing with PBS, all slices were stained with 4',6diamidino-2-phenylindole (DAPI, $5 \mu \mathrm{g} \mathrm{mL}^{-1}$, Sigma) to reveal the nuclei. Images were acquired by a confocal laser scanning microscope. Relative fluorescence intensity was analyzed with ImageJ software.

\section{Two-photon excitation fluorescence (TPEF) and second harmonic generation (SHG) imaging of the MSCS-CHS/ADM scaffold transplanted into the wound site}

Mice were anesthetized and disinfected with alcohol to dissect the cutaneous wound repair areas after treatment with the MSCs-ADM and MSCs-CHS/ADM scaffolds after $7,14,21$, and 28 days. Finally, the samples were flattened and placed on clean glass slides. These tissue samples were imaged by two photon laser scanning confocal microscope (TPLSCM). Collagen in ADM or CHS/ ADM scaffold was imaged by SHG signals with the emission wavelength of 405-415 nm (central wavelength of $410 \mathrm{~nm}$ ) at a laser excitation wavelength of $820 \mathrm{~nm}$. The regenerated wound areas in each sample were continuously scanned (a frame of a 2D image) for collagen and green fluorescence protein (GFP) simultaneously at a speed of $6 \mathrm{~s}$ per frame. To identify whether the selected tissue samples were viable, the exposure time of the samples was strictly limited to within $30 \mathrm{~min}$. The intensities of the GFP and SHG signals were analyzed using ImageJ software.

\section{Real-time quantitative PCR analysis}

Total RNA from the wound samples and single cell suspensions was extracted with an RNA extraction kit (AIVD, Guangzhou, China). Then, cDNA synthesis was performed using a PrimeScript ${ }^{\mathrm{T} M} \mathrm{RT}$ reagent Kit with gDNA Eraser (TaKaRa, Dalian, China). The RT-PCR primer sequences were synthesized by Sangon Biotech (Shanghai, China) in Table 1:

PCR was conducted on a TaKaRa Real-Time PCR machine using SYBR Green $\left(\mathrm{SYBR}^{\odot}\right.$ Premix Ex $\mathrm{Taq}^{\mathrm{Ts}}$, TaKaRa, Beijing, China) and validated with a CFX96 RealTime PCR system (Applied Biosystems). The mRNA expression level of the target gene was normalized to $\beta$-actin expression and was analyzed using the comparative method of relative quantification $\left(2^{-\Delta \Delta} \mathrm{Ct}\right)$. Five repetitions were performed on each sample.

\section{Western blotting}

Protein lysates from the wound samples were homogenized in RIPA buffer containing protease inhibitors and denatured at $95^{\circ} \mathrm{C}$ in sample buffer for 5-10 min. Each sample protein was electrophoresed on $12 \%$ SDS-PAGE and then transferred to PVDF membranes. The membranes were blocked in buffer (5\% nonfat dry milk, 10 $\mathrm{mM}$ Tris, $100 \mathrm{mM} \mathrm{NaCl}, 0.1 \%$ Tween-20) at $4{ }^{\circ} \mathrm{C}$ overnight and incubated with rabbit polyclonal GFP antibody (1:1500 dilution, Abcam) and mouse monoclonal $\beta$-actin antibody (1:2000 dilution, Affinity) at $4{ }^{\circ} \mathrm{C}$ overnight. After washing, the membranes were incubated with secondary antibodies at room temperature for $2 \mathrm{~h}$ and washed with TBST three times for $10 \mathrm{~min}$ each time. The detection of the western blot signals was performed

Table 1 Primer sequences

\begin{tabular}{ll}
\hline Gene Forward primer (5'-> 3') & Reverse primer (5'-> 3') \\
\hline -actin CGTTGACATCCGTAAAGACC & TAGGAGCCAGAGCAGTAATC \\
GFP GCACGACTTCTTCAAGTCCGCCAT & GCGGATCTTGAAGTTCACCTTGAT \\
GCC & GCC \\
LPL AGTTTGACCGCCTTCCGCGG & TCCTGTCACCGTCCATCCATGGA \\
PPARY ACTGCCGGATCCACAAAA & TCTCCTTCTCGGCCTGTG \\
ALP AACCCAGACACAAGCATTCC & CCAGCAAGAAGAAGCCTTTTG \\
Runx-2 TGCCACCTCTGACTTCTGCC & CGCTCCGGCCCACAATCTC \\
\hline
\end{tabular}


with an ODYSSEY Infrared Imaging System (LI-COR) and the generated signals were analyzed using ImageJ software.

\section{Immunofluorescence and histological analysis}

Mice from each group were photographed on different days after different wounding days (7, 14, 21, and 28). The percent wound closure was calculated by Image-Pro Plus software and quantified with the follow equation:

Wound closure rate $(\%)=$ (initial wound area - indicated wound area)/initial wound area $\times 100 \%$.

In addition, paraffin sections of wound tissue were autoclaved for antigen retrieval and blocked with a peroxide blocking agent and normal goat serum (Beyotime, Guangzhou, China). The samples were incubated with primary antibodies to green fluorescence protein (GFP, $1: 1000$, Sigma) and vascular smooth muscle actin ( $\alpha$ SMA, 1:1000, Millipore Chemicon) for double-labeled immunofluorescence staining, as well as $\alpha$-SMA for single-labeled immunofluorescence staining. After washing, the samples were incubated with the following secondary antibodies: CY3-labeled goat anti-rabbit IgG (for GFP, 1:300, Abcam) and FITC-labeled goat anti-rat IgG (for $\alpha$-SMA, 1:300, Abcam). All slices were stained with 4',6-diamidino-2-phenylindole (DAPI, Sigma) to show the nuclei. Images were acquired by confocal laser scanning microscopy. Masson's trichome staining was used to analyze the regeneration of the collagen accumulation state with ImageJ. In addition, hematoxylin and eosin (H\&E) staining was carried out for the histological analysis of wound regeneration. All data were analyzed using ImageJ software.

\section{Statistics analysis}

All statistical results are presented as the means \pm SD. Statistical analyses were performed with OriginPro 8.5 (OriginLab, USA), and significant differences were assessed with one-way ANOVA and Dunnett's multiple comparison tests, with statistical significance levels denoted by a single asterisk $(p<0.05)$, two asterisks $(p<$ $0.01)$, or three asterisks $(p<0.001)$, and are indicated in each figure.

\section{Results and discussion}

Chitosan enhances the survival activity of MSCs under ROS microenvironment

An in vitro coculture was performed to evaluate the survival ability of MSCs with chitosan under ROS microenvironment. Hydrogen peroxide $\left(\mathrm{H}_{2} \mathrm{O}_{2}\right)$ was used to imitate ROS environment that could lead to induce oxidative stress damage in MSCs. As shown in Fig. 1a, MSC viability was inhibited by $\mathrm{H}_{2} \mathrm{O}_{2}$ in a dosedependent manner compared with the control. These data reveal that ROS inhibits MSC viability and results in MSC death. Once the concentration of $\mathrm{H}_{2} \mathrm{O}_{2}$ was above $30 \mu \mathrm{M}$, the survival of MSCs was apparently reduced; therefore, 30- $\mu \mathrm{M}$ concentration was selected and applied in the following detriment investigation of $\mathrm{H}_{2} \mathrm{O}_{2}$. Next, the antioxidant activity of the chitosan on MSC viability under $\mathrm{H}_{2} \mathrm{O}_{2}$-treated $(30 \mu \mathrm{M})$ was investigated and the $\mathrm{H}_{2} \mathrm{O}_{2}$-untreated was used as the control group. The introduction of chitosan recovered the ROSreduced MSC survival rate in a dose-dependent manner, especially when the chitosan concentration was $1 \mathrm{mg} / \mathrm{ml}$ (Fig. 1b, c). Likewise, in the assay of cell-matrix adhesion, while the adhesive MSCs were reduced to $37.8 \pm$ $1.37 \%$ of control by $\mathrm{H}_{2} \mathrm{O}_{2}$ treatment $(30 \mu \mathrm{M})(* * p<0.01)$, the addition of $1 \mathrm{mg} / \mathrm{ml}$ chitosan could recover the reduced MSC adhesion induced by ROS (Fig. 1d). Also, as Fig. 1e demonstrates, the incorporation of $1 \mathrm{mg} / \mathrm{ml}$ chitosan significantly decreases the level of intracellular ROS in $\mathrm{H}_{2} \mathrm{O}_{2}$ environment. Therefore, the CHS/ADM scaffold with the $1 \mathrm{mg} / \mathrm{ml}$ concentration of the chitosan was chosen for the next experiment.

The effect of chitosan on differentiation capability of MSCs is then to be explored, which is crucial because it is one of the most significant characteristics of MSCs for their application in regenerative medicine. By staining adipocytes and osteoblasts with Oil Red $\mathrm{O}$ and Alizarin red, respectively, chitosan was confirmed to have no significant influence on differentiation of MSCs (Fig. S1a). Further experiments showed that $\mathrm{H}_{2} \mathrm{O}_{2}$ decreased the adipogenic and/or osteogenic differentiation, while chitosan could effectively prevent the $\mathrm{H}_{2} \mathrm{O}_{2}$-induced decrease (Fig. S1b).

\section{The characterization of the CHS/ADM scaffold and its effect on the survival and differentiation of MSCs}

FT-IR spectra from ADM, chitosan (CHS), and CHS/ ADM are shown in Fig. 2a. The spectra of ADM demonstrate four characteristic absorption bands at 3321, 1640, 1553 , and $1238 \mathrm{~cm}^{-1}$. Generally, amide I bands $(1640$ $\mathrm{cm}^{-1}$ ) originate from $\mathrm{C}=\mathrm{O}$ stretching vibrations coupled to $\mathrm{N}-\mathrm{H}$ bending vibrations. The amide II bands (1553 $\mathrm{cm}^{-1}$ ) arise from the N-H bending vibrations coupled to $\mathrm{C}-\mathrm{N}$ stretching vibrations. The amide III band (1238 $\mathrm{cm}^{-1}$ ) is the combination peak between N-H deformation and $\mathrm{C}-\mathrm{N}$ stretching vibrations. The vibrations of hydroxyl group, $-\mathrm{OH}$, appear at $3306 \mathrm{~cm}^{-1}$ [44]. The spectra of chitosan contain six characteristic absorption bands. The vibrations of $-\mathrm{OH}$ and free $-\mathrm{NH}_{2}$ appear at 3455 and $3364 \mathrm{~cm}^{-1}$, respectively. The absorption bands at 1648,1592 , and $1381 \mathrm{~cm}^{-1}$ are amide I, amide II, and $\mathrm{NH}_{2}$ bending and $\mathrm{C}-\mathrm{O}$ stretching of primary alcohol groups, respectively. The last one, at $1098 \mathrm{~cm}^{-1}$, represents $\mathrm{C}-\mathrm{O}-\mathrm{C}$ glycosidic linkage between chitosan monomers [45-47]. FT-IR spectra of CHS/ADM illustrate similar characteristic peaks of the parent molecules, 

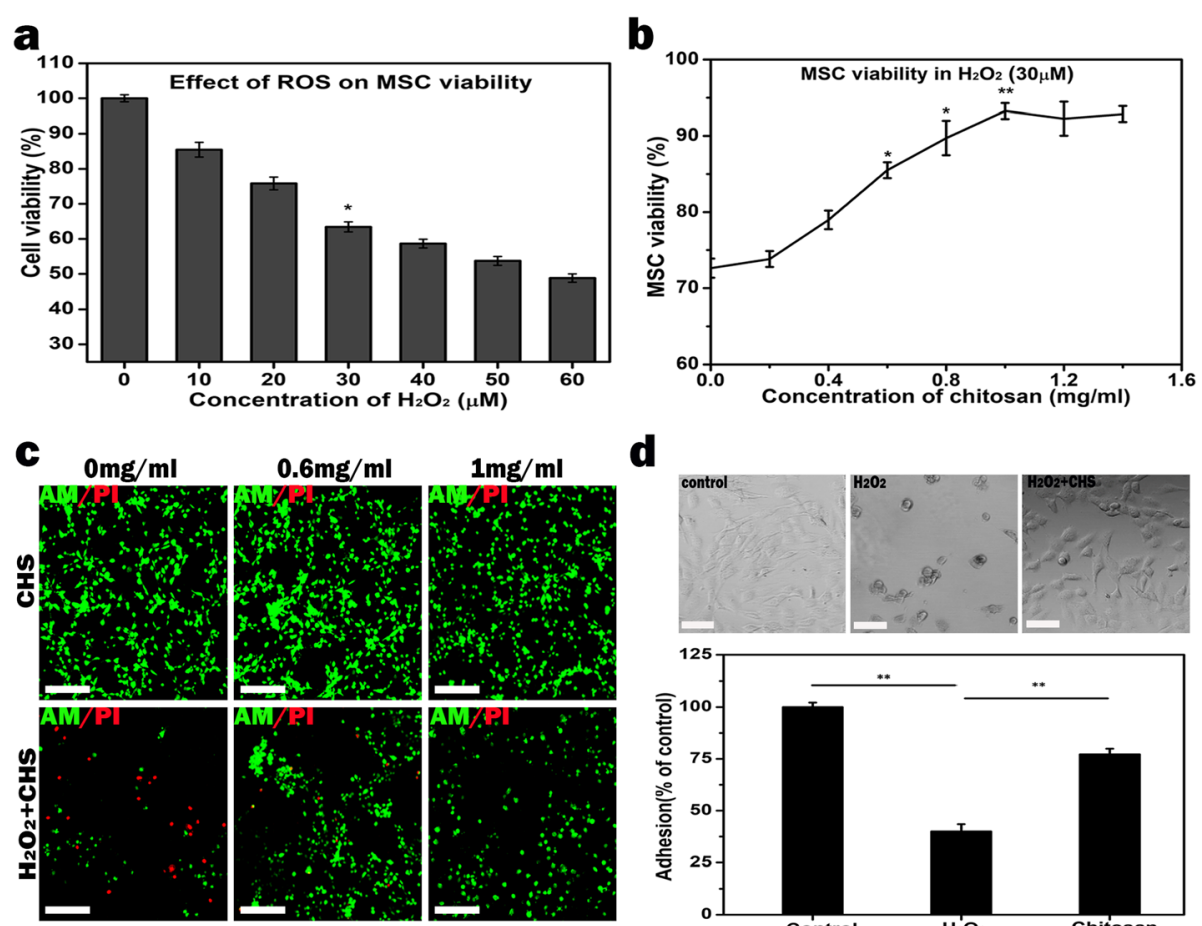

$0.6 \mathrm{mg} / \mathrm{ml}$

$1 \mathrm{mg} / \mathrm{ml}$

d
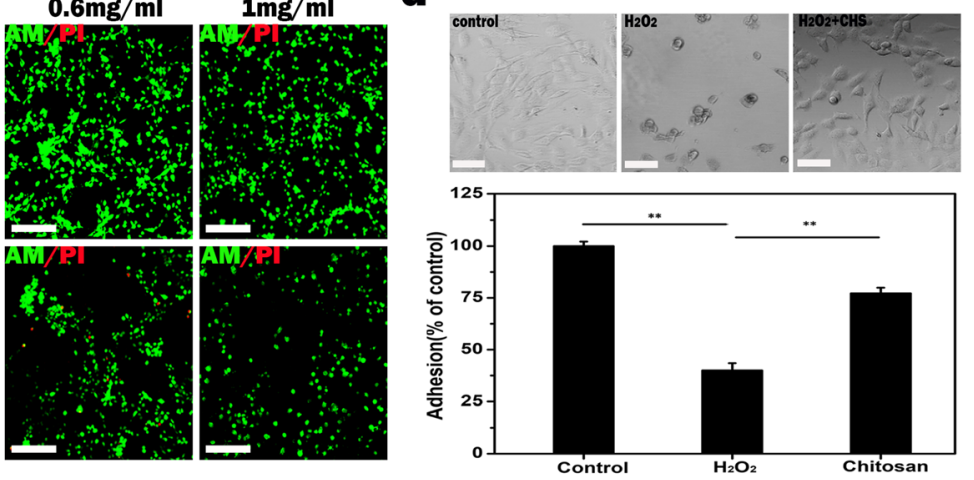

e
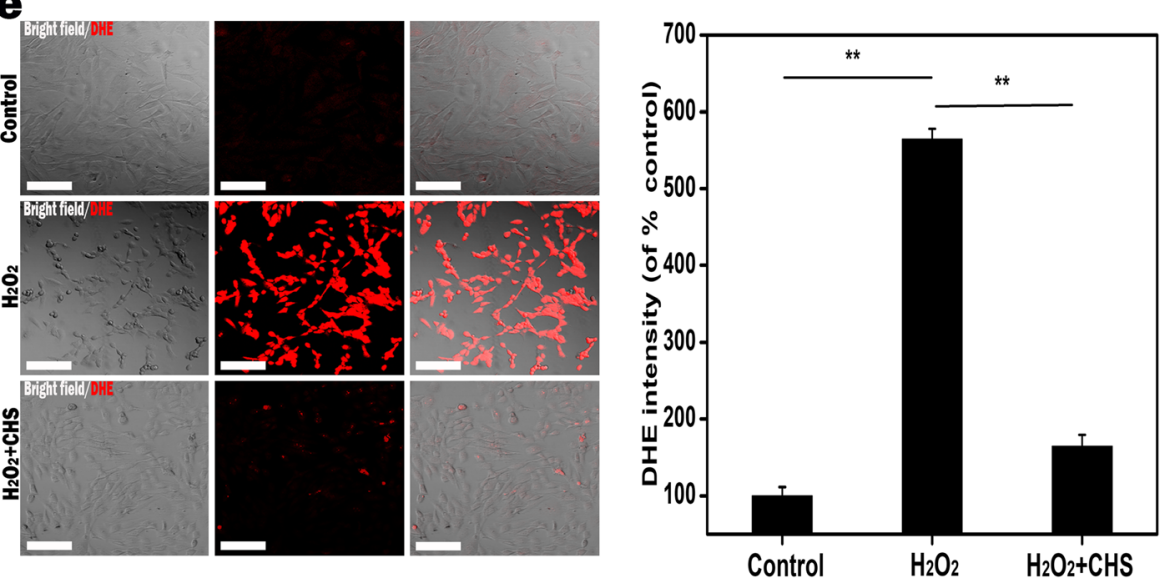

Fig. 1 ROS scavenging capacity of the chitosan. a ROS inhibits MSC viability in a dose-dependent manner. $\mathbf{b}$ Various chitosan concentrations restore the reduced viability of MSCs in a dose-dependent manner. c Live/dead staining images of the MSCs with $\mathrm{H}_{2} \mathrm{O}_{2}$ treatment. Green and red represent lived cells and dead cells, respectively. Scale bars are $200 \mu \mathrm{m}(n=5)$. $\mathbf{d}$ Images of adhesive cells on culture plastic surface in presence of $\mathrm{H}_{2} \mathrm{O}_{2}$. Quantification of adhesive MSCs shows that ROS significantly inhibits the adhesion of MSCs compared to control (untreated with $\mathrm{H}_{2} \mathrm{O}_{2}$ ), while chitosan significantly attenuates the effect induced by ROS. e The measurement of intracellular ROS by DHE staining. Control indicates untreated with $\mathrm{H}_{2} \mathrm{O}_{2}, \mathrm{H}_{2} \mathrm{O}_{2}$ indicates $\mathrm{H}_{2} \mathrm{O}_{2}$ treatment, and $\mathrm{H}_{2} \mathrm{O}_{2}+\mathrm{CHS}$ indicates $\mathrm{H}_{2} \mathrm{O}_{2}$ +chitosan treatment; scale bars are $200 \mu \mathrm{m}^{*}{ }^{*} p<0.01,{ }^{*} p<0.05$

hence indicating that collagen and chitosan interactions are polyelectrolytic with oppositely charged ionic polymers, particularly the cationic group of chitosan $\left(-\mathrm{NH}_{3}{ }^{+}\right)$and negative group in anionic collagen $\left(-\mathrm{COO}^{-}\right)$[47-49]. The degradation degree of the ADM and CHS/ADM scaffolds is also shown in Fig. 2b. The results illustrate that both scaffolds displayed an increasing enzymatic degradation with time increasing. After $96 \mathrm{~h}$, the degradation mass of the ADM scaffold was approximately $94.12 \pm 3.37 \%$, but the CHS/ADM scaffolds had a lower degradation rate $(78.75 \pm 3.17 \%)$, which indicates that the degradation rate of ADM scaffold becomes slower with the introduction of chitosan. The lower degradation rate in CHS/ADM scaffold is not exactly known, but it could be that the chitosan blocks collagenase binding sites in collagen and/or directly inhibits enzymatic activity of collagenase, thereby enhancing the resistance to degradation of collagen [50].

As shown in Fig. 2c-inset, The CHS/ADM scaffolds reveal looser than the ADM scaffolds. This was also 

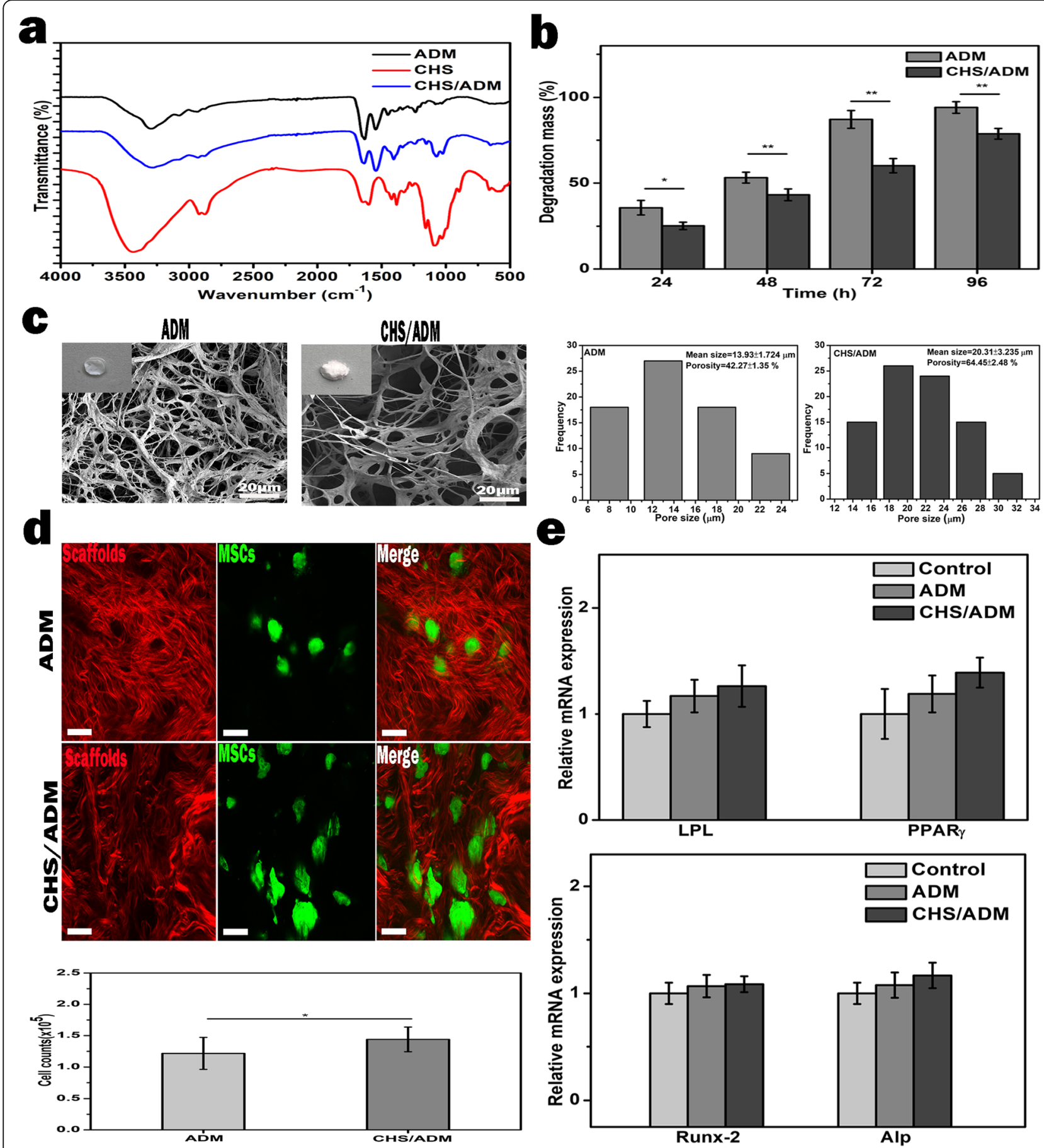

Fig. 2 The characterization of the CHS/ADM scaffold and its effect on the survival and differentiation of MSCs. a FT-IR absorption spectra of the CHS, ADM, and CHS/ADM scaffolds. $\mathbf{b}$ The percent degradation mass of the ADM and CHS/ADM scaffolds after enzymatic degradation at different time points $\left({ }^{* *} p<0.01,{ }^{*} p<0.05, n=3\right)$. c SEM images showing the morphologies of the ADM and CHS/ADM scaffolds. The corresponding illustrations show 8-mmdiameter ADM and CHS/ADM scaffolds. Scale bar, $20 \mu \mathrm{m}$. The corresponding pore size and porosity distribution of the ADM and CHS/ADM scaffolds are shown right $(n=3)$. $\mathbf{d}$ TPEF-SHG imaging and cell counting of MSCs cultured on the ADM and CHS/ADM scaffolds for $24 \mathrm{~h}(n=3)$. Scale bar, $10 \mu \mathrm{m}$. $\mathbf{e}$ Quantitative analysis of the mRNA expression levels of specific adipocyte genes (LPL, PPARY) and osteogenic genes (Runx-2, ALP) of MSCS seeded on either the ADM and CHS/ADM scaffolds or only in medium as the control on days 14 and 21 
demonstrated from the scanning electron microscopy images in Fig. 2c. The results show that the ADM scaffold comprised a collagen fiber network structure with a limited pore size $(13.93 \pm 1.724 \mu \mathrm{m})$ and porosity $(42.27 \pm 1.35 \%)$. In contrast, the CHS/ADM scaffold exhibited a network structure of chitosan-coupled collagen fiber with an optimized pore size $(20.31 \pm 3.235 \mu \mathrm{m})$ and porosity (64.45 $\pm 2.48 \%$ ) (Fig. 2c, right). This bigger pore size in CHS/ADM scaffold is possibly mainly caused by rehydration and relyophilization process in the refreezedrying treatment in the preparation of the CHS/ ADM scaffold. This refreeze-drying procedure may lead to some collagen fibers in the ADM scaffold being combined with each other to form a sheet-like structure. As a result, the pore size is increased correspondingly [51, 52]. In addition, the content and the concentration of chitosan may improve the structural stability of the matrices in CHS/ADM scaffold which is related to the porosity $[53,54]$. This bigger pore size and higher porosity might improve the binding capacity of the MSC [54].

Next, two-photon excitation fluorescence (TPEF) and second harmonic generation (SHG) imaging was performed to observe the morphological characteristics of $\mathrm{GFP}^{+}$-MSCs seeded on the scaffolds in real time (Fig. 2d). As expected, the MSCs seeded on the CHS/ADM scaffold showed more survival activity than that in ADM scaffold. Quantitatively, MSCs after 1-day seeding on the ADM and CHS/ADM scaffolds were counted by the cell counting plate after trypan blue staining. As shown in Fig. 2d, the number of the MSCs in CHS/ ADM scaffold group is more than that in ADM scaffold, which indicates that CHS/ADM scaffold has desirable properties for MSCs survival. Additionally, the differentiation capability of the MSCs seeded on the scaffolds was explored; there was no significant difference in both adipocyte and osteoblast differentiation induction between CHS/ADM scaffold and those in ADM scaffold in Fig. 2e. The above data reveal that the CHS/ADM scaffold has appropriate structural characteristics and excellent biocompatibility, which is favorable as a potential candidate scaffold for long-term stem cell therapy.

\section{CHS/ADM scaffold enhances the survival activity of MSCs under ROS microenvironment}

In Fig. 3a (above), the results of the live/dead assay showed that most cells were stained green, and only a few dead cells (blue) could be observed in the CHS/ ADM scaffold after $\mathrm{H}_{2} \mathrm{O}_{2}$ treatment. And the MSC viability (\%) by CCK8 assay shown in Fig. 3a (below) in the $\mathrm{CHS} / \mathrm{ADM}$ scaffold is also better than that in ADM scaffold after $\mathrm{H}_{2} \mathrm{O}_{2}$ treatment. These data indicate that the CHS/ADM scaffold could improve the survival of MSCs in the ROS microenvironment compared with the ADM scaffold group. Furthermore, dihydroethidium (DHE) staining was performed to observe the level of intracellular ROS in MSCs-ADM or MSCs-CHS/ADM scaffolds in Fig. 3b. The results revealed that the level of intracellular ROS in ADM scaffold group significantly increased in $\mathrm{H}_{2} \mathrm{O}_{2}$ environment. In comparison, this increase in ROS was partially eliminated by the CHS/ADM scaffolds. These data revealed that the detrimental effects displayed by ROS could be evidently attenuated by the introduction of chitosan into the ADM scaffold via the scavenging of ROS. Most importantly, the levels of ROS are markedly lower in the CHS/ADM scaffold compared to the ADM scaffold, which further reduces MSC death caused by oxidative damage and strengthens the survival capacity of MSCs in the ROS microenvironment during wound healing.

\section{The CHS/ADM scaffold attenuates the inflammatory response and intracellular ROS at the wound site}

Neutrophils are a central component of the inflammatory response, which have the potential to cause severe tissue destruction by releasing an excess ROS onto host tissues $[16,55,56]$. It is essential to evaluate the effects of CHS/ADM scaffold on neutrophil and particularly focus on the amount of ROS. As inflammatory response usually occurs at the first few days of injury and neutrophils are short lived, they may disintegrate and disappear after 2 or 3 days. Therefore, in order to understand the neutrophil infiltration and ROS production level during inflammatory response, the first days like day 1,3 , and 5 after injury were chosen as the time points for investigation. In the present study, immunohistochemical analysis of sections was performed by labeling with CD11b to identify the infiltrated neutrophils during the inflammation period. On day 1 after the operation, neutrophils were present in a moderate amount in the CHS/ADM scaffold group and subsequently decreased over time (Fig. 4a). However, significant amounts of neutrophils were observed in the ADM scaffold group during this period. As such, the CD11b intensity in the ADM scaffold group was obviously higher than that in the CHS/ ADM scaffold group during the same period (* $p<0.05)$. These data demonstrate that the CHS/ADM scaffold alleviated the inflammatory response, which is beneficial for providing a favorable survival environment for implanted stem cells. The higher amount of infiltrated neutrophils in ADM scaffold are most likely due to collagen degradation, which is initially induced by recruited neutrophils because of injury to vascularized connective tissue through releasing collagenase [18]. As collagen degradation-derived products might directly serve as chemotactic stimuli for neutrophils in vivo $[16,57]$, the peptide fragments of collagen produced by the degradation of the ADM scaffold consequently lead to excessive neutrophil infiltration. 


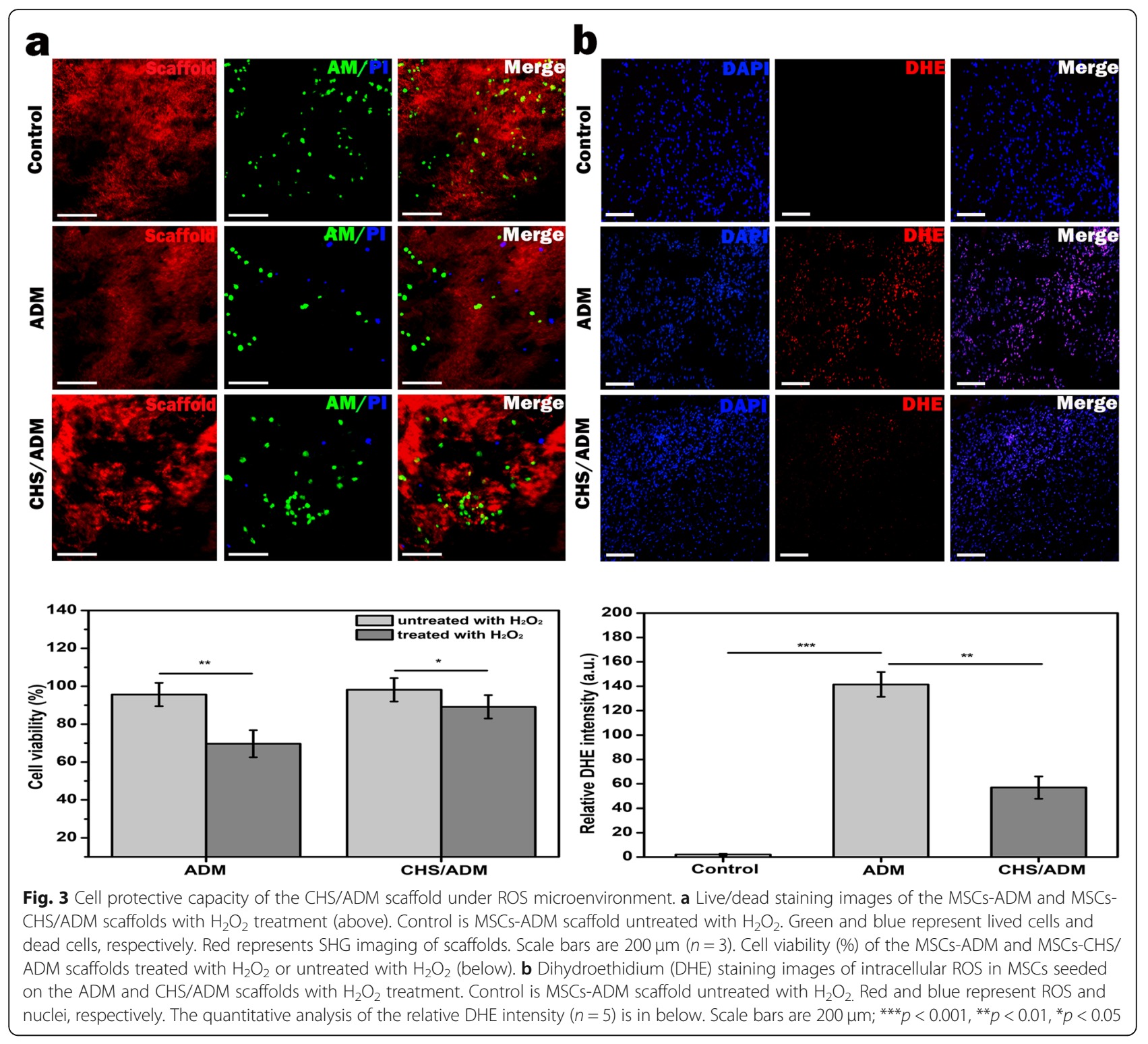

Simultaneously, immunofluorescence with dihydroethidium (DHE) staining was performed to explore the intracellular ROS in the MSCs seeded on scaffolds after transplantation at the wound site. In Fig. 4b, strong red fluorescence can be observed around/in the cell nucleus (blue) in ADM scaffold at day 3, revealing more production of ROS compared to the control group. This overproduction of ROS in ADM scaffold is likely closely associated with the higher amount of infiltrated neutrophils in inflammation, as a high concentration of ROS is confirmed usually being released by neutrophils to stunt the growth of adjacent bacteria in wound environment [58]. Also, there is evidence that a continuous recruitment of inflammatory cells serves as a substantial source of ROS [59]. However, the CHS/ADM scaffold played an effective ROS scavenging role that contributed to a reduction in the red fluorescence during the same period. Moreover, the relative DHE intensity in the CHS/ADM scaffold group was apparently lower than that in the ADM scaffold group after 3 days ( $" * p<0.01)$, which is probably due to the introduction of chitosan in the composite scaffold that reduces ROS in the environment. These data show that the CHS/ADM scaffold attenuates the inflammatory response and reduces the amount of ROS in the environment.

\section{The CHS/ADM scaffold enhances MSC retention in wound} healing

Retention is the duration of survival. To reveal the retention of stem cells implicates determining their time of death. In order to analyze the ability of the CHS/ ADM scaffold in improving implanted stem cell 


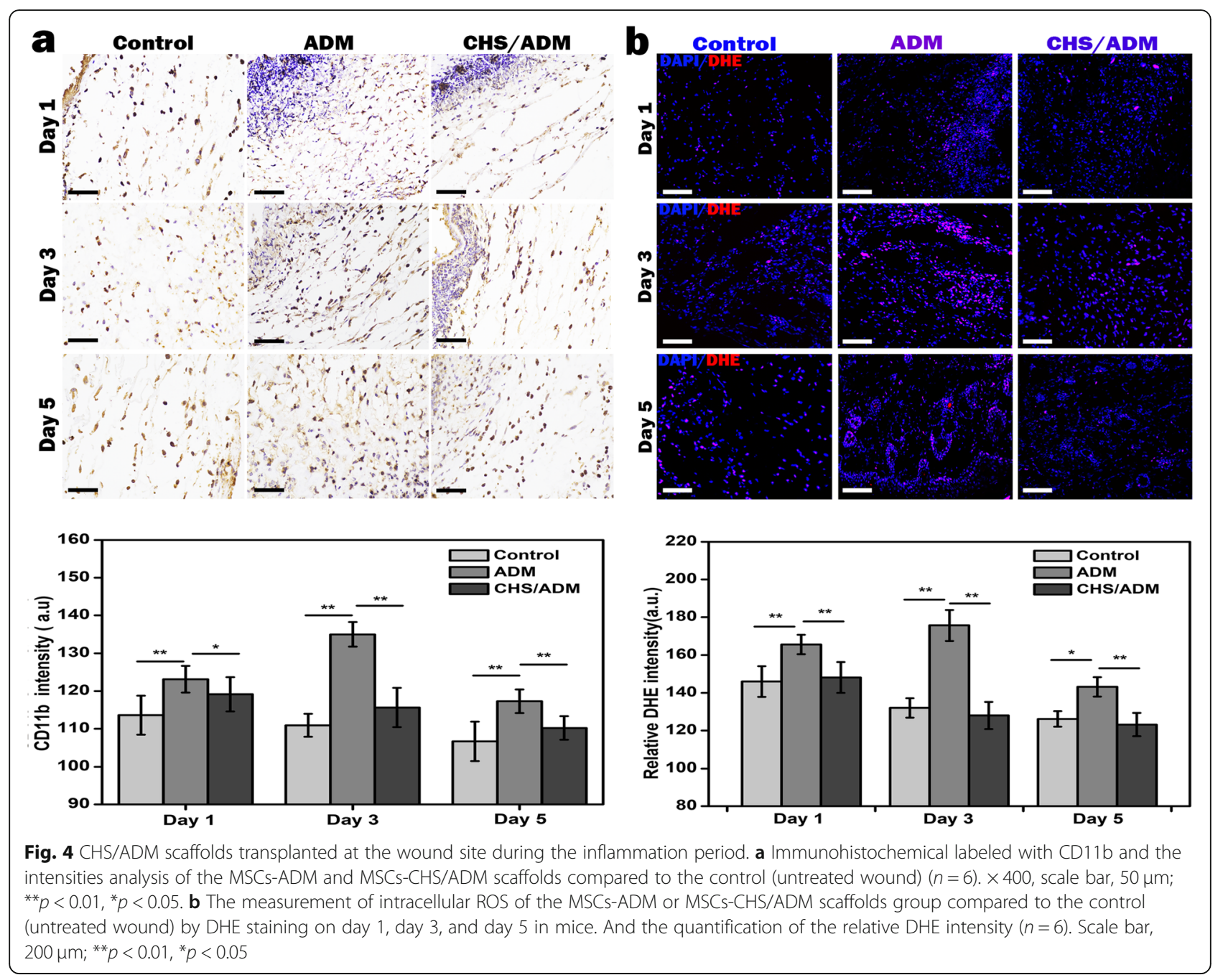

retention in wound repair, TPEF-SHG imaging was therefore performed on later days $7,14,21$, and 28 after the transplantation of the MSCs-CHS/ADM scaffolds into skin wounds. At the same time, MSCs-ADM scaffolds were used as the comparison group. As shown in Fig. $5 \mathrm{a}$ and $\mathrm{b}$, the amount of $\mathrm{GFP}^{+}-\mathrm{MSCs}$ in the CHS/ ADM scaffold group was significantly higher than that in the ADM scaffold group at the same time period. Moreover, a dramatic decrease in cell viability (green) was observed on day 14 after the operation in the ADM scaffold group. Through evaluation of the GFP and SHG signal intensities, the changes in the number of $\mathrm{GFP}^{+}$MSCs and collagen accumulation were depicted at different time points, as shown in Fig. $5 \mathrm{c}$ and $\mathrm{d}$. In addition, as the treatment time increased, the newborn collagen content of both scaffolds obviously increased, and the collagen content of the CHS/ADM scaffold group was higher than that of the ADM scaffold group. It is worth noting that cells delivered in CHS/ADM scaffolds displayed markedly more $\mathrm{GFP}^{+}$-MSCs than that of
ADM scaffolds on the same healing day, and $\mathrm{GFP}^{+}$MSCs retained till day 28 in the CHS/ADM scaffold group, while under ADM scaffold, $\mathrm{GFP}^{+}$-MSCs disappeared after 14 days of implantation. These results were further confirmed by PCR and western blot analyses (Fig. 5e, f). The mRNA and protein expression levels of GFP in the CHS/ADM scaffold group were markedly higher than those in the ADM scaffold group during the same time period. The level of GFP decreased in the wounds over time and downregulated to zero till day 28, which is much longer than the day in ADM group (about 14 days). These results suggest that the CHS/ ADM scaffold delivery system effectively enhanced MSC retention in the hostile wound microenvironment over 3 weeks, which is advisable for wound healing.

\section{Evaluation of angiogenesis response}

Angiogenesis at the later stage of wound healing is generally used to evaluate the effect of wound healing. Angiogenesis was evaluated in sections of regenerated 
a

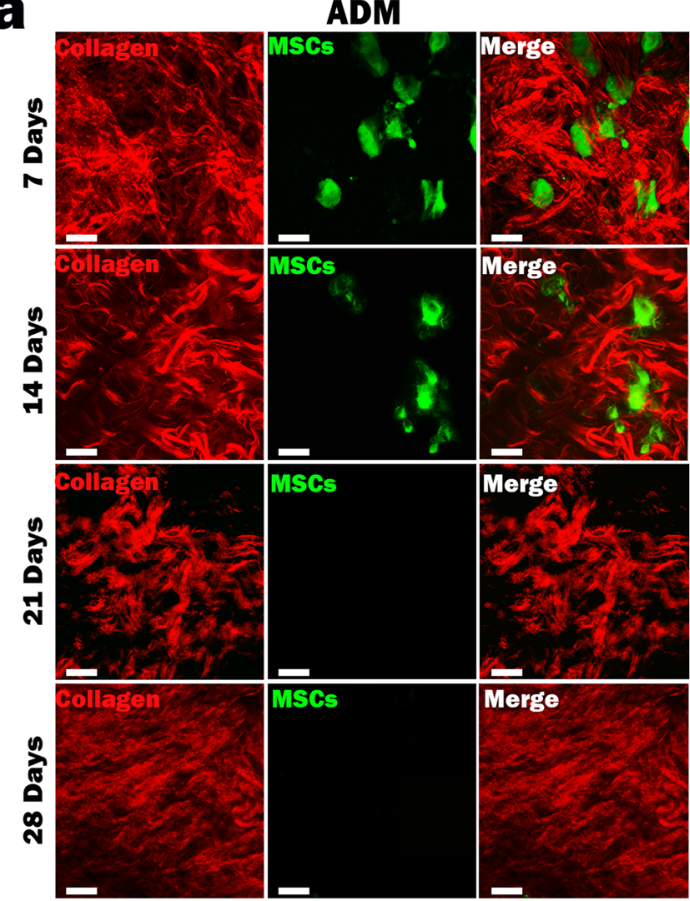

$c$
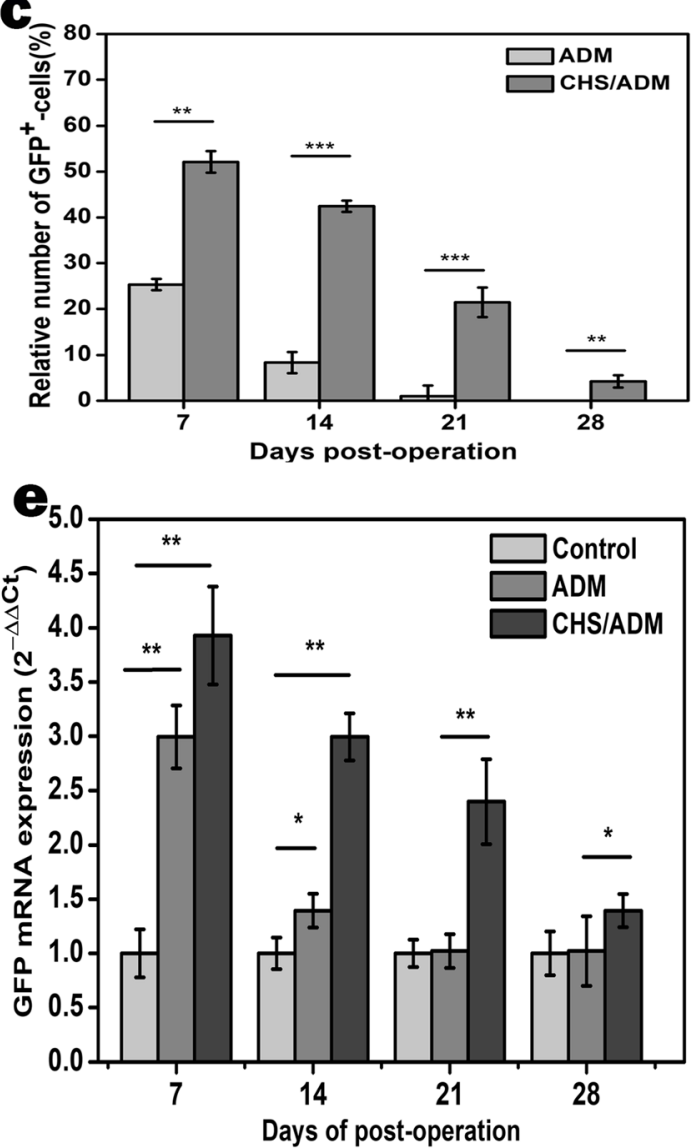

b
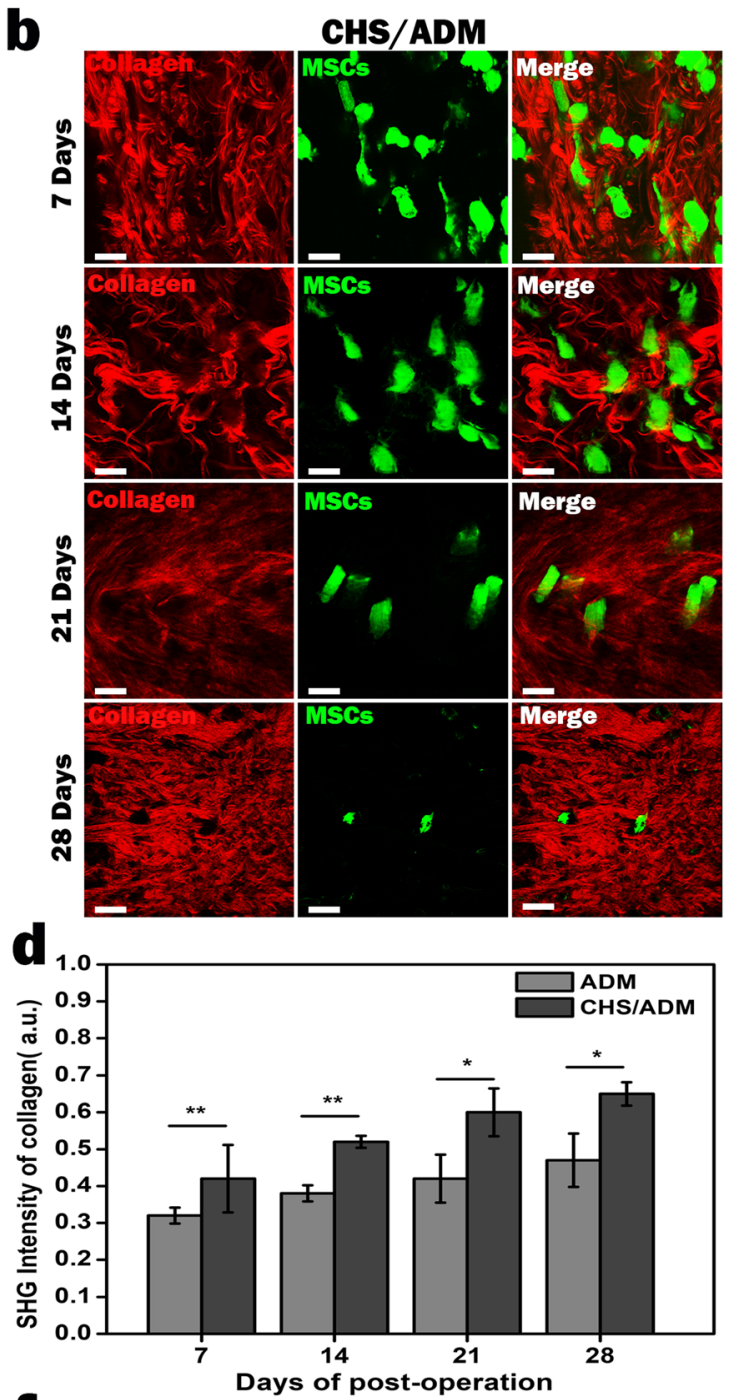

f

ADM

CHS/ADM
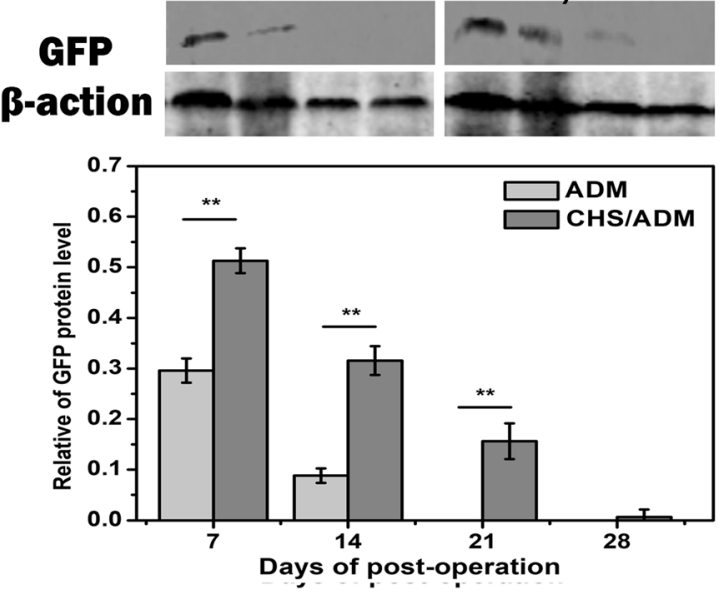

Fig. 5 (See legend on next page.) 
(See figure on previous page.)

Fig. 5 The CHS/ADM scaffold enhanced MSC retention in vivo. b TPEF-SHG imaging of MSCs that seeded on the CHS/ADM scaffolds transplanted into the wound site on days 7, 14, 21, and 28. Green and red represent GFP+-MSCs and collagen, respectively. a ADM scaffold as the comparison group (original magnification, $\times 60$; scale bar, $10 \mu \mathrm{m}$ ). $\mathbf{c}$ The change in the number of MSCs in the wounds on days 7, 14, 21 , and 28 after transplantation. $\mathbf{d}$ SHG intensity of collagen at different time points with increased treatment in the ADM and CHS/ADM scaffold groups. e Comparison of GFP levels in each group (7, 14, 21, and 28 days) via PCR and $\mathbf{f}$ western blot analysis. Statistical analysis of the expression of GFP. Data are expressed as the mean values, and the error bars represent the SD $(n=5) .{ }^{* *} p<0.001,{ }^{* *} p<0.01,{ }^{*} p<0.05$

tissue at the wound sites by double-immunofluorescence staining with GFP and alpha-smooth muscle actin $(\alpha-$ SMA). The control group was only stained with $\alpha$-SMA antibody (Fig. 6a). As the angiogenesis usually occurs later on 7 days, therefore, the later days like days 7,14 , 21 , and 28 in wound healing were chosen as the time points for investigation. The results show that the wounds in the CHS/ADM scaffold group appeared to have substantially more $\mathrm{GFP}^{+}$-MSCs and $\alpha$-SMA fluorescence than the untreated control and ADM scaffold groups at the same treatment day (Fig. 6a-c). The survival of the implanted $\mathrm{GFP}^{+}$-MSCs was quantified by the fluorescence intensity of the cells in the assay (Fig. 6b). The results further demonstrate that the retention time of $\mathrm{GFP}^{+}$-MSCs in the CHS/ADM scaffold group was extended, and few MSCs were detected on day 28.

In addition, alpha-smooth muscle actin ( $\alpha$-SMA) staining of wound sections from different treatment groups were performed to identify the vasculature with smooth muscle cells. Angiogenesis networks mainly appeared on day 14 in the CHS/ADM scaffold group, and annular mature vessels principally appeared after 21 days. Quantitative analysis indicated that the blood vessel density of the CHS/ADM scaffold group $\left(231.08 \pm 46 \mathrm{~mm}^{2}\right)$ was significantly higher than that of the ADM scaffold group $\left(214.87 \pm 21 \mathrm{~mm}^{2}\right)$ and control group $\left(185.05 \pm 25 \mathrm{~mm}^{2}\right)$ (" $p<0.05$ ) (Fig. 6c). Furthermore, the vessel diameter of the CHS/ADM scaffold group was larger than that of
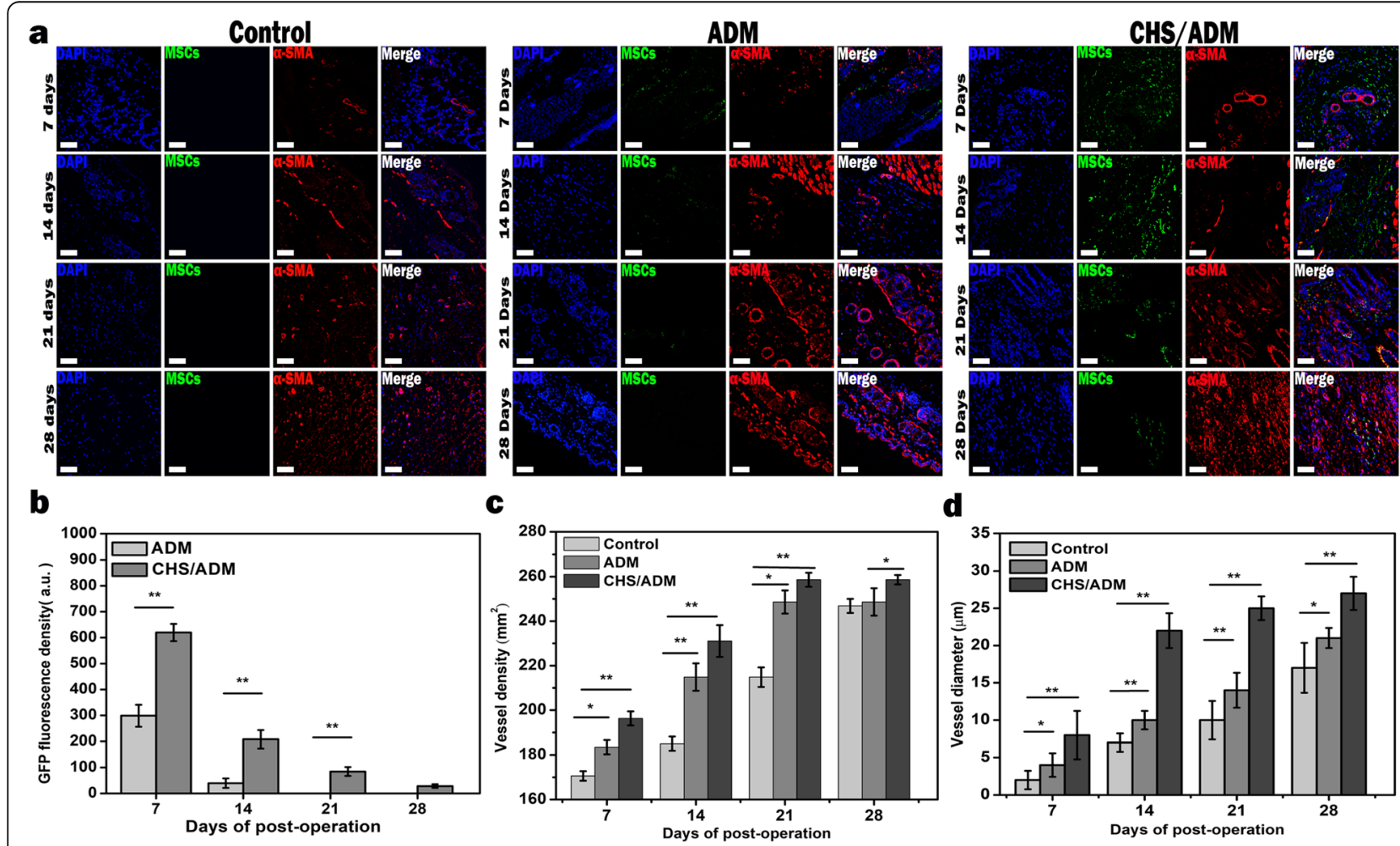

d

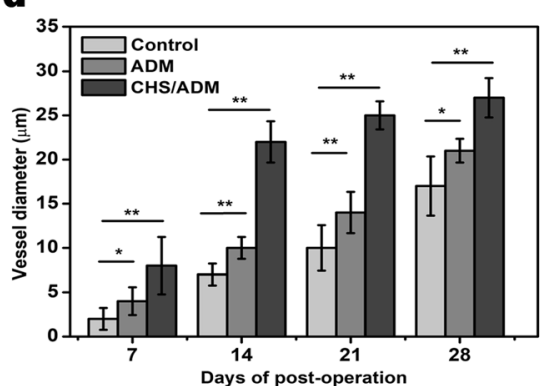

Fig. 6 The occurrence of vascular regeneration at the wound site. a Fluorescence imaging of wound tissues in the control, MSCs-ADM scaffold, and MSCs-CHS/ADM scaffold groups on days 7, 14, 21, and 28. Green, red, and blue represent GFP+-cells, a-SMA-cells, and nuclei, respectively. Scale bar, $200 \mu \mathrm{m}$. b Fluorescence intensities of GFP with different treatment times. Quantitative analysis of c the vessel density and $\mathbf{d}$ diameter of the MSCs-ADM scaffold, MSCs-CHS/ADM scaffold and control groups on days 7, 14, 21, and 28. Data are expressed as the mean values and SD $(n=5) .{ }^{* *} p<0.01,{ }^{*} p<0.05$ 


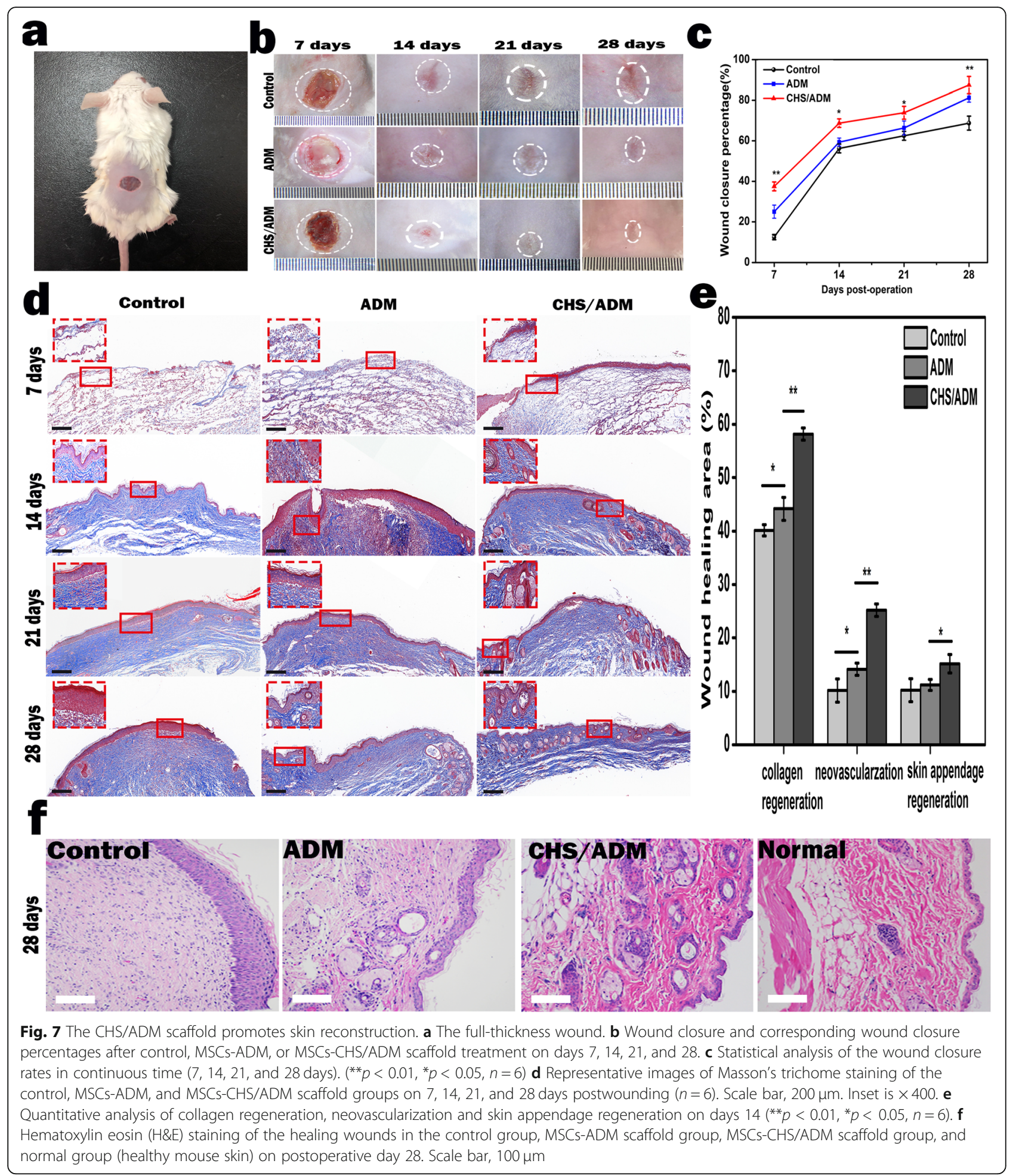

the $\mathrm{ADM}$ and control groups in the healing tissues $(23.54 \pm 4.17 \mathrm{~mm}, 13.54 \pm 3.13 \mathrm{~mm}$, and $7.53 \pm 2.34 \mathrm{~mm}$, respectively) (Fig. 6d). These data indicate that MSCs delivered in the CHS/ADM scaffold enhance vessel growth in wounds.
Transplantation of CHS/ADM scaffold accelerates murine wound healing

An 8-mm-diameter full-thickness murine excisional wound healing model was performed to evaluate the therapeutic efficacy of the MSCs seeded on CHS/ 
ADM scaffolds in vivo (Fig. 7a). The ADM and CHS/ ADM scaffold treatment groups showed obvious acceleration of wound closure at different levels compared with the control group (Fig. 7b, c). Notably, the CHS/ADM scaffold group exhibited better wound closure than the ADM scaffold group on the same postoperative day. Moreover, the wound area completely closed after 14 days, and almost no scar was found after 21 days in the CHS/ADM scaffold group $(" p<0.05)$. However, the wound was almost closed with a scab covering in the control group after 28 days. The Masson-stained sections showed that the transplanted MSCs in the CHS/ADM scaffold group had mature bundles of collagen deposition, while the control and ADM scaffold groups had disorganized collagen (Fig. 7d). Subsequently, collagen regeneration, neovascularization, and skin appendage regeneration were analyzed after 14 days (Fig. 7e), and the wounds treated with CHS/ADM scaffolds showed obviously more favorable than other groups. Moreover, hematoxylin eosin (H\&E) staining indicated that the CHS/ADM scaffold group after 28 days appeared to be more similar to that of normal skin tissue than the control and ADM scaffold groups (Fig. 7f). Overall, these data demonstrate that MSCs delivered by CHS/ADM scaffolds markedly accelerated wound closure, reduced scar formation, and promoted wound repair.

\section{Conclusion}

In summary, we developed a novel chitosan/acellular dermal matrix scaffold, which has favorable biodegradability and desirable biocompatibility, and particularly acts as an excellent ROS scavenger to address the challenge of transplanted cell survival by eliminating ROS during the inflammatory response. This scaffold provides a protective environment for cell survival in vivo and contributes to the vessel growth and the acceleration of wound healing, which demonstrates a great potential use in wound healing and other tissue regeneration applications.

\section{Supplementary information}

Supplementary information accompanies this paper at https://doi.org/10. 1186/s13287-020-01901-6.

Additional file 1: Figure S1. The effect of chitosan on MSCs differentiation without $(n=6)$ and with $\mathrm{H}_{2} \mathrm{O}_{2}(30 \mu \mathrm{M})(n=5)$. Oil Red $\mathrm{O}$ staining of adipocytes and Alizarin red staining of osteoblasts at day 14 and 21, respectively. Scar bar: 20 $\mu$ m; ${ }^{* *} p<0.01,{ }^{*} p<0.05$. Figure S2. SEM images showing the morphologies of the ADM and CHS/ADM scaffolds after $24 \mathrm{~h}$ degradation by collagenase. Scar bar: $10 \mu \mathrm{m}(\mathrm{n}=5)$. Figure S3. Young's modulus of the ADM and CHS/ADM scaffolds ( $n=6)$. Figure S4. The percent degradation mass of the ADM and CHS/ADM scaffolds after enzymatic degradation at different time points under ROS environment $\left(30 \mu \mathrm{M} \mathrm{H}_{2} \mathrm{O}_{2}\right)(\mathrm{n}=5)$.

\section{Abbreviations}

ROS: Reactive oxygen species; CHS: Chitosan; CHS/ADM: Chitosan/acellular dermal matrix; MSCs: Mesenchymal stem cells; ADM: Acellular dermal matrix; ECM: Extracellular matrix; SEM: Scanning electron microscopy;

PMNs: Polymorphonuclear neutrophils; DHE: Dihydroethidium; Calcein-AM/ PI: Calcein-AM/propidium iodide; DAPI: 4',6-Diamidino-2-phenylindole; PBS: Phosphate-buffered saline; RT-PCR: Real-time polymerase chain reaction; TPEF: Two-photon excitation fluorescence; SHG: Second harmonic generation; TPLSCM: Two photon laser scanning confocal microscope; GFP: Green fluorescence protein; SDS-PAGE: Polyacrylamide gel electrophoresis; LI-COR: Infrared Imaging System; a-SMA: Ascular smooth muscle actin; H\&E: Hematoxylin and eosin

\section{Acknowledgements}

The authors give special thanks to the Guangzhou Institutes of Biomedicine and Health, Chinese Academy of Sciences for supplying the TPEF-SHG microscopy instrumentation.

\section{Authors' contributions}

Xiaoyuan D. and W.L. designed the studies. W.L., Danyang L., and Baoping L. synthesized and characterized materials. Xiaoyang Q. performed TPEF-SHG microscopy. W.L. performed in vitro and vivo experiments. W.L. and Heting C. analyzed and evaluated the animal results. All authors participated in writing and discussions and have given approval to the final version of the manuscript.

\section{Funding}

This study was supported by the National Natural Science Foundation of China (Nos. 81671729 and 81171379) and the Guangzhou Municipal Science and Technology Project of China (No. 201804010051).

\section{Availability of data and materials}

Not applicable.

\section{Ethics approval and consent to participate}

All animal experiments conducted in this research were performed in accordance with the guidelines of South China Normal University Intramural Animal Use and Care Committee and the NIH guidelines for the care and use of laboratory animals were met.

\section{Consent for publication}

Not applicable.

\section{Competing interests}

The authors declare that they have no competing interests.

\section{Author details}

${ }^{1}$ MOE Key Laboratory of Laser Life Science, College of Biophotonics \& Institute of Laser Life Science, South China Normal University, Guangzhou 510631, China. ${ }^{2}$ Guangdong Provincial Key Laboratory of Laser Life Science, College of Biophotonics, South China Normal University, Guangzhou 510631, China. ${ }^{3}$ The Brain Cognition and Brain Disease Institute of Shenzhen Institutes of Advanced Technology, Chinese Academy of Sciences, Shenzhen 518055, China. ${ }^{4}$ Guangzhou Institutes of Biomedicine and Health, Chinese Academy of Sciences, Guangzhou 510530, China.

Received: 24 January 2020 Revised: 13 August 2020 Accepted: 24 August 2020 Published online: 07 September 2020

\section{References}

1. Dong Y, Rodrigues M, Li X, Kwon SH, Kosaric N, Khong S, Gao Y, Wang W, Gurtner GC. Injectable and tunable gelatin hydrogels enhance stem cell retention and improve cutaneous wound healing. Advanced Functional Materials. 2017:27:24

2. Burdick JA, Mauck RL, Gerecht S. To serve and protect: hydrogels to improve stem cell-based therapies. Cell Stem Cell. 2016;18:1.

3. Ringe J, Kaps C, Burmester GR, Sittinger M. Stem cells for regenerative medicine: advances in the engineering of tissues and organs. Naturwissenschaften. 2002;89:8 
4. Birgersdotter A, Sandberg R, Ernberg I. Gene expression perturbation in vitro--a growing case for three-dimensional (3D) culture systems. Semin Cancer Biol. 2005;15:5.

5. Liu Z, Tang M, Zhao J, Chai R, Kang J. Looking into the future: toward advanced 3D biomaterials for stem-cell-based regenerative medicine. Adv Mater. 2018;30:17.

6. Chattopadhyay S, Raines RT. Review collagen-based biomaterials for wound healing. Biopolymers. 2014;101:8.

7. Cross VL, Zheng Y, Won Choi N, Verbridge SS, Sutermaster BA, Bonassar LJ, Fischbach C, Stroock AD. Dense type I collagen matrices that support cellular remodeling and microfabrication for studies of tumor angiogenesis and vasculogenesis in vitro. Biomaterials. 2010;31:33.

8. Wang Q, Jin Y, Deng X, Liu H, Pang H, Shi P, Zhan Z. Second-harmonic generation microscopy for assessment of mesenchymal stem cell-seeded acellular dermal matrix in wound-healing. Biomaterials. 2015;53:659-68.

9. Han X, Liu H, Chen M, Gong L, Pang H, Deng X, Jin Y. Acellular dermal matrix from one-day-old mouse skin on adult scarless cutaneous wound repair by second harmonic generation microscopic imaging. RSC Adv. 2016;6:76.

10. Gurtner GC, Werner S, Barrandon Y, Longaker MT. Wound repair and regeneration. Nature. 2008;453:7193.

11. Guo S, Dipietro LA. Factors affecting wound healing. J Dent Res. 2010;89:3.

12. Eming SA, Krieg T, Davidson JM. Inflammation in wound repair: molecular and cellular mechanisms. J Invest Dermatol. 2007:127:3.

13. Menke NB, Ward KR, Witten TM, Bonchev DG, Diegelmann RF. Impaired wound healing. Clin Dermatol. 2007;25:1.

14. Wong SL, Demers M, Martinod K, Gallant M, Wang Y, Goldfine AB, Kahn CR, Wagner DD. Diabetes primes neutrophils to undergo NETosis, which impairs wound healing. Nat Med. 2015;21:7.

15. Patil PS, Leipzig ND. Fluorinated methacrylamide chitosan sequesters reactive oxygen species to relieve oxidative stress while delivering oxygen. J Biomed Mater Res A. 2017;105:8.

16. Laskin DL, Kimura T, Sakakibara S, Riley DJ, Berg RA. Chemotactic activity of collagen-like polypeptides for human peripheral blood neutrophils. J Leukoc Biol. 1986;39:3.

17. Monboisse JC, Bellon G, Dufer J, Randoux A, Borel JP. Collagen activates superoxide anion production by human polymorphonuclear neutrophils. Biochem J. 1987;246:599-603.

18. Weiss SJ, Peppin G, Ortiz X, Ragsdale C, Test ST. Oxidative autoactivation of latent collagenase by human neutrophils. Science. 1985;227:4688.

19. Martin P, Leibovich SJ. Inflammatory cells during wound repair: the good, the bad and the ugly. Trends Cell Biol. 2005;15:11.

20. Kaplan SS, Basford RE, Jeong MH, Simmons RL. Mechanisms of biomaterial-induced superoxide release by neutrophils. J Biomed Mater Res. 1994;28:3.

21. Ley K, Laudanna C, Cybulsky MI, Nourshargh S. Getting to the site of inflammation: the leukocyte adhesion cascade updated. Nat Rev Immunol. 2007:7:9.

22. Kolaczkowska E, Kubes P. Neutrophil recruitment and function in health and inflammation. Nat Rev Immunol. 2013;13:3.

23. de Oliveira S, Rosowski EE, Huttenlocher A. Neutrophil migration in infection and wound repair: going forward in reverse. Nat Rev Immunol. 2016;16:6.

24. Nathan C. Neutrophils and immunity: challenges and opportunities. Nat Rev Immunol. 2006;6:3.

25. Martin P. Wound healing--aiming for perfect skin regeneration. Science. 1997;276:5309

26. Dovi JV, He LK, DiPietro LA. Accelerated wound closure in neutrophildepleted mice. J Leukoc Biol. 2003;73:4.

27. Selders GS, Fetz AE, Radic MZ, Bowlin GL. An overview of the role of neutrophils in innate immunity, inflammation and host-biomaterial integration. Regen Biomater. 2017;4:1.

28. Henson PM, Vandivier RW. The matrix degrades, neutrophils invade. Nat Med. 2006;12:3.

29. Schafer M, Werner S. Oxidative stress in normal and impaired wound repair. Pharmacol Res. 2008;58:2

30. Lee SH, Gupta MK, Bang JB, Bae H, Sung HJ. Current progress in reactive oxygen species (ROS)-responsive materials for biomedical applications. Advanced Healthcare Materials. 2013;2:6.

31. Tapeinos C, Larrañaga A, Sarasua JR, Pandit A. Functionalised collagen spheres reduce $\mathrm{H}_{2} \mathrm{O}_{2}$ mediated apoptosis by scavenging overexpressed ROS. Nanomedicine. 2018;7:2397-405.
32. Gailit JA, Colflesh DA, Rabiner IL, Simone JA, Goligorsky MS. Redistribution and dysfunction of integrins in cultured renal epithelial cells exposed to oxidative stress. Am J Physiol-Renal Physiol. 1993;264:1.

33. Shi C, Zhu Y, Ran X, Wang M, Su Y, Cheng T. Therapeutic potential of chitosan and its derivatives in regenerative medicine. J Surg Res. 2006;133:2.

34. Liu Z, Wang H, Wang Y, Lin Q, Yao A, Cao F, Li D, Zhou J, Duan C, Du Z, Wang $Y$, Wang $C$. The influence of chitosan hydrogel on stem cell engraftment, survival and homing in the ischemic myocardial microenvironment. Biomaterials. 2012;33:11.

35. Ma L. Collagen/chitosan porous scaffolds with improved biostability for skin tissue engineering. Biomaterials. 2003;24:26.

36. Altman AM, Yan Y, Matthias N, Bai X, Rios C, Mathur AB, Song Y-H, Alt EU. IFATS collection: human adipose-derived stem cells seeded on a silk fibroinchitosan scaffold enhance wound repair in a murine soft tissue injury model. Stem Cells. 2009:27:1.

37. Esumi K, Takei N, Yoshimura T. Antioxidant-potentiality of gold-chitosan nanocomposites. Colloids Surf B: Biointerfaces. 2003:32:2.

38. Kim I-Y, Seo S-J, Moon H-S, Yoo M-K, Park I-Y, Kim B-C, Cho C-S. Chitosan and its derivatives for tissue engineering applications. Biotechnol Adv. 2008;26:1.

39. Ueno H, Yamada H, Tanaka I, Kaba N, Matsuura M, Okumura M, Fujinaga T. Accelerating effects of chitosan for healing at early phase of experimental open wound in dogs. Biomaterials. 1999;20:15.

40. Tamer $T$, Aacute $K$, Mohyeldin M, Soltes L. Free radical scavenger activity of chitosan and its aminated derivative. J Appl Pharmaceutical Sci. 2016;6(04): 195-201.

41. Mi FL, Tan YC, Liang HF, Sung HW. In vivo biocompatibility and degradability of a novel injectable-chitosan-based implant. Biomaterials. 2002:23:1

42. Chiu YL, Chen SC, Su CJ, Hsiao CW, Chen YM, Chen HL, Sung HW. pHtriggered injectable hydrogels prepared from aqueous N-palmitoyl chitosan: in vitro characteristics and in vivo biocompatibility. Biomaterials. 2009;30:28.

43. Muzzarelli RA. Biochemical significance of exogenous chitins, chitosan in animals, patients. Carbohydr Polym. 1993;20:7-16.

44. Taravel MN, Domard A. Collagen and its interaction with chitosan: II. Influence of the physicochemical characteristics of collagen. Biomaterials. 1995;16:11.

45. Sionkowska A, Wisniewski M, Skopinska J, Kennedy CJ, Wess TJ. The photochemical stability of collagen-chitosan blends. J Photochem Photobiol A Chem. 2004;162:2-3.

46. Tangsadthakun C, Kanokpanont S, Sanchavanakit N, Pichyangkura R, Banaprasert T, Tabata Y, Damrongsakkul S. The influence of molecular weight of chitosan on the physical and biological properties of collagen/ chitosan scaffolds. J Biomaterials Sci Polymer Edition. 2007;18:2.

47. Horn MM, Martins VCA, de Guzzi Plepis AM. Interaction of anionic collagen with chitosan: effect on thermal and morphological characteristics. Carbohydr Polym. 2009;77:2.

48. Chen Z, Mo X, He C, Wang H. Intermolecular interactions in electrospun collagen-chitosan complex nanofibers. Carbohydr Polym. 2008;72:3.

49. Taravel MN, Domard A. Relation between the physicochemical characteristics of collagen and its interactions with chitosan: I. Biomaterials. 1993;14:12.

50. Kishen A, Shrestha S, Shrestha A, Cheng C, Goh C. Characterizing the collagen stabilizing effect of crosslinked chitosan nanoparticles against collagenase degradation. Dental Materials. 2016;32:8.

51. Zhu Y, Liu T, Song K, Jiang B, Ma X, Cui Z. Collagen-chitosan polymer as a scaffold for the proliferation of human adipose tissue-derived stem cells. J Mater Sci Mater Med. 2009;20:3.

52. Lie, Gao C, Mao Z, Shen J, Hu X, Han C. Thermal dehydration treatment and glutaraldehyde cross-linking to increase the biostability of collagen-chitosan porous scaffolds used as dermal equivalent. J Biomaterials Sci Polymer Edition. 2003;14:8

53. Peng L, Cheng XR, Wang JW, Xu DX, Wang GE. Preparation and evaluation of porous chitosan/collagen scaffolds for periodontal tissue engineering. J Bioact Compat Polym. 2006;21:3.

54. Kobayashi SD, Voyich JM, DeLeo FR. Regulation of the neutrophil-mediated inflammatory response to infection. Microbes Infect. 2003;5:14.

55. Butler J, Rocker GM, Westaby S. Inflammatory response to cardiopulmonary bypass. Ann Thoracic Surg. 1993;55:2.

56. Haslett C. Granulocyte apoptosis and its role in the resolution and control of lung inflammation. Am J Respiratory Critical Care Med. 1999; 160(supplement_1):S5-S11. 
57. Stecher VJ. The chemotaxis of selected cell types to connective tissue degradation products. Ann N Y Acad Sci. 1975;256:177-89.

58. Dunnill C, Patton T, Brennan J, Barrett J, Dryden M, Cooke J, Georgopoulos NT. Reactive oxygen species (ROS) and wound healing: the functional role of ROS and emerging ROS-modulating technologies for augmentation of the healing process. Int Wound J. 2017;14:1.

59. Kim YW, Byzova TV. Oxidative stress in angiogenesis and vascular disease. Blood J Am Soc Hematol. 2014;123:5.

\section{Publisher's Note}

Springer Nature remains neutral with regard to jurisdictional claims in published maps and institutional affiliations.

Ready to submit your research? Choose BMC and benefit from:

- fast, convenient online submission

- thorough peer review by experienced researchers in your field

- rapid publication on acceptance

- support for research data, including large and complex data types

- gold Open Access which fosters wider collaboration and increased citations

- maximum visibility for your research: over $100 \mathrm{M}$ website views per year

At $\mathrm{BMC}$, research is always in progress.

Learn more biomedcentral.com/submissions 